\title{
Molecular epidemiology and recombination of Enterovirus A71 in mainland China from 1987 to 2017
}

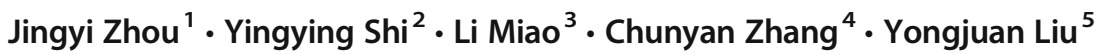

Received: 13 October 2020 / Revised: 2 February 2021 / Accepted: 11 February 2021 / Published online: 19 February 2021

(C) Springer Nature Switzerland AG 2021

\begin{abstract}
Enterovirus A71 (EV-A71) is an important pathogen of severe hand, foot, and mouth disease (HFMD) in young children. This study aimed to retrospectively analyze the molecular epidemiology and recombination of EV-A71 in mainland China during 1987-2017. Phylogenetic tree showed that besides the previously reported subgenotypes A, B5, C0, C2, C3, and C4, a new subgenotype C6 emerged in mainland China. Recombination analysis indicated that C4 EV-A71 was derived from a common ancestor as a "double-recombinant" virus by intertypic recombination between C EV-A71 and CVA4, CVA5, CVA14, and CVA16 strains in $\mathrm{P} 3$ region and intratypic recombination between $\mathrm{C}$ and B EV-A71 strains in P2 region. The B5 EV-A71 shared high similarity with C EV-A71 in P1 region while it contained an unidentified sequence in P2 and P3 regions with two possible recombination patterns: one occurred between C4 EV-A71 and CVA3, CVA5, CVA6, CVA10, and CVA12 stains with one breakpoint in 3C, and the other occurred between C1, C2, C3, and C5 EV-A71 and CVA4, CVA5, CVA14, and CVA16 strains with two breakpoints in the $2 \mathrm{~A} / 2 \mathrm{~B}$ junction and $3 \mathrm{C}$. The $\mathrm{C} 2 \mathrm{EV}-\mathrm{A} 71$ was probably a recombinant virus between $\mathrm{C} 4 \mathrm{EV}-\mathrm{A} 71$ and CVA8 strains with two breakpoints located in the 5'UTR and 2A/2B junction. Moreover, an incredible recombination of C6 EVA71 occurred between C4 and C2 EV-A71 with multiple breakpoints. Thus, continuous studies on EV-A71 genome characteristics are still useful and essential for monitoring emergence of new viruses and preventing HFMD outbreaks.
\end{abstract}

Keywords Enterovirus A71 $\cdot$ Molecular epidemiology $\cdot$ Recombination $\cdot$ Phylogenetic tree

\section{Introduction}

Hand, foot, and mouth disease (HFMD) is a common infectious disease in young children that often caused by Enterovirus A species (EV-A) (Zhang et al., 2011). The EVA species includes 25 serotypes viruses such as the

Yongjuan Liu

lyjquanzhiyuan_09@163.com

1 Department of Anorectal Surgical, Lianyungang No. 1 People's Hospital, Lianyungang 222000, People's Republic of China

2 Department of Immunology, Jianghan University, Wuhan 430056, People's Republic of China

3 Department of Pediatrics Internal Medicine, Lianyungang No. 1 People's Hospital, Lianyungang 222000, People's Republic of China

4 Department of Clinical Laboratory, Lianyungang No. 1 People's Hospital, Lianyungang 222000, People's Republic of China

5 Department of Central Laboratory, Lianyungang No. 1 People's Hospital, Tongguan Road No. 182, Haizhou District, Lianyungang 222000, People's Republic of China coxsackievirus A group (CVA2-CVA8, CVA10, CVA12, CVA14, and CVA16), human enteroviruses (EV-A71, EVA76, EV-A89-EV-A91, EV-A114, EV-A119-EV-A121), and non-human enteroviruses (EV-A92, EV-A122-EVA125) (Simmonds et al., 2020). HFMD is mostly a kind of mild and self-limited disease, but some cases infected by EVA71 can rapidly develop serious neurological complications and even death (Chen et al., 2019). Since 2007, EV-A71 has caused several large outbreaks of HFMD in the Western Pacific Region, especially in mainland China. Although EVA71 vaccine has been put on the market since 2016, EV-A71 is still an important pathogen of severe and fatal HFMD (Zhao, 2019). No effective and specific antiviral drugs are available. Severe HFMD caused by EV-A71 remains an important public health problem for children.

As a member of EV-A in the genus Enterovirus of the family Picornaviridae, EV-A71 contains a single-stranded, positive-sense RNA genome of nearly $7.4 \mathrm{~kb}$ in length (Lin et al., 2017). The genome of EV-A71 consists of 5'- and 3'untranslated regions (UTR) and one large open reading frame (ORF). The ORF encodes a large polyprotein divided into 3 
regions, $\mathrm{P} 1, \mathrm{P} 2$, and $\mathrm{P} 3$. The $\mathrm{P} 1$ region encodes 4 structural proteins VP1-VP4, which constitutes the virus capsid, while the $\mathrm{P} 2$ and $\mathrm{P} 3$ regions encode 7 non-structural proteins $2 \mathrm{~A}-2 \mathrm{C}$ and $3 \mathrm{~A}-3 \mathrm{D}$, which participate in polyprotein processing, viral replication, and interactions with the host (Wang and $\mathrm{Li}$, 2019).

Based on phylogenetic analysis of VP1 gene, EV-A71 is currently classified into 8 genotypes A-H (Deshpande et al., 2003; Saxena et al., 2015; Bessaud et al., 2014; Majumdar et al., 2018). Genotype A contained a sole prototype strain $\mathrm{BrCr}$ isolated in California, USA, in 1969 (Schmidt et al., 1974) and some strains emerged in China in 2008-2010 (Vakulenko et al., 2019). The emergence of genotype A EVA71 strains in China was considered as a release of the prototype strain from a laboratory into circulation (Vakulenko et al., 2019). Genotypes $B$ and $C$ are subdivided into subgenotypes B0-B5 and C0-C5, respectively (Noisumdaeng et al., 2018). In the Asia-Pacific region, subgenotypes $\mathrm{C} 1, \mathrm{C} 2, \mathrm{~B} 3, \mathrm{~B} 4$, and C4 prevailed in Australia, subgenotypes B3, B4, B5, C1, and C2 prevailed in Malaysia and Singapore, subgenotypes B2, $\mathrm{C} 1, \mathrm{C} 2, \mathrm{~B} 4, \mathrm{C} 4$, and B5 prevailed in Japan, and subgenotypes B4, C2, C4, B5, and C5 prevailed in Taiwan (Mizuta et al., 2014). Different from these areas with the alternative circulation of several predominant subgenotypes, $\mathrm{C} 4$ was the sole predominant subgenotype in mainland China (Zhang et al., 2013). New genotypes were identified in India (genotypes D and G) (Deshpande et al., 2003; Saxena et al., 2015), subSaharan Africa (genotype E) (Bessaud et al., 2014), Madagascar (genotype F) (Bessaud et al., 2014), and Pakistan (genotype H) (Majumdar et al., 2018). EV-A71 shared high genetic diversity in developing countries where the circulating genotypes might be underestimated. Except for $\mathrm{C} 4$, genotype A and subgenotypes B5, C2, and C3 had emerged in mainland China (Zhang et al., 2013; Tao et al., 2012; Li et al., 2018; Zhang et al., 2009). This study aimed to know whether there were new genotypes or subgenotypes in mainland China by analyzing the phylogenetics of EV-A71 based on a large dataset of VP1 sequences available from GenBank database. And the genomic characteristics of EVA71 strains belonging to different subgenotypes were analyzed by recombination analyses.

\section{Materials and methods}

\section{Viral sequences}

This study involved no human participants or human experimentation. The complete VP1 gene sequences of 3037 Chinese EV-A71 strains covering the time period 19872017 were downloaded from the GenBank database. The VP1 sequences of 10 international EV-A71 and CVA16 prototype strains were used as the reference sequences. Three thousand forty-eight VP1 sequences were used in phylogenetic analysis (Table S1). Moreover, the genome sequences of 6 EV-A71 strains whose isolated date was the latest in each subgenotype (C4a1, C4a2, C4b, B5, C2, and C6) were selected as the query sequences for recombination analysis. And another 13 Chinese EV-A71 strains, 10 international EVA71 strains, and 11 CVA prototype strains (CVA2-8, CVA10, CVA12, CVA14, and CVA16) were used for comparison in recombination analysis. Forty genome sequences were employed in recombination analysis (Table S2).

\section{Phylogenetic analysis}

The VP1 gene sequences were converted into a merged file in Clustal format by SeqVerter ${ }^{\mathrm{TM}}$. Then, the merged file was converted into MEGA format by MEGA-X. Phylogenetic tree was constructed by MEGA-X using the neighbor-joining method (bootstrap test 1000 replicates) with Kimura 2parameter model.

\section{Recombination analysis}

The merged sequence file was first aligned by MEGA-X with ClustalW method to generate a nucleotide alignment. The similarity plot and bootscan analysis were performed by SimPlot3.5.1. The Kimura 2-parameter distance model and the neighbor-joining tree model were selected in all bootscanning with a window size of 200 nucleotides moving along the alignment in 20-bp increments.

\section{Results}

\section{Phylogenetic analysis of the entire VP1 gene of EV- A71 in mainland China}

The phylogenetic tree showed that except for 3011 strains clustered into subgenotype C4, 17 strains clustered into genotype A, 4 strains clustered into subgenotype B5, 2 strains clustered into subgenotype $\mathrm{C} 2,2$ orphan strains respectively clustered into subgenotype $\mathrm{C} 0$ and $\mathrm{C} 3$, and one EV-A71 strain DL71 (GenBank accession number KF982854) clustered into a new branch in genotype C (Fig. 1). The strain DL71 was isolated from Dalian City in Liaoning Province in 2012. It did not fall into any of the six known subgenotypes $\mathrm{C} 0-\mathrm{C} 5$ of EVA71 but formed a separate genetic branch containing no other strains. The phylogenetic trees based on VP4 and 2A sequences also indicated that DL71 belonged to a previously unknown subgenotype in genotype C (Fig. S1). The new branch was defined as subgenotype C6. The phylogenetic analyses indicated that 7 genotypes or subgenotypes including $\mathrm{A}, \mathrm{B} 5, \mathrm{C} 0, \mathrm{C} 2, \mathrm{C} 3, \mathrm{C} 4$, and $\mathrm{C} 6$ of EV-A71 circulated in mainland China. 
Fig. 1 Phylogenetic analysis of VP1 gene sequences of EV-A71 with reference strains. The evolutionary branches were colored differently by the viral isolated time. Clade $\mathrm{C} 4 \mathrm{a} 2$ of subgenotype C4 was compressed to save space. The scale bar indicated the number of nucleotide substitutions per site

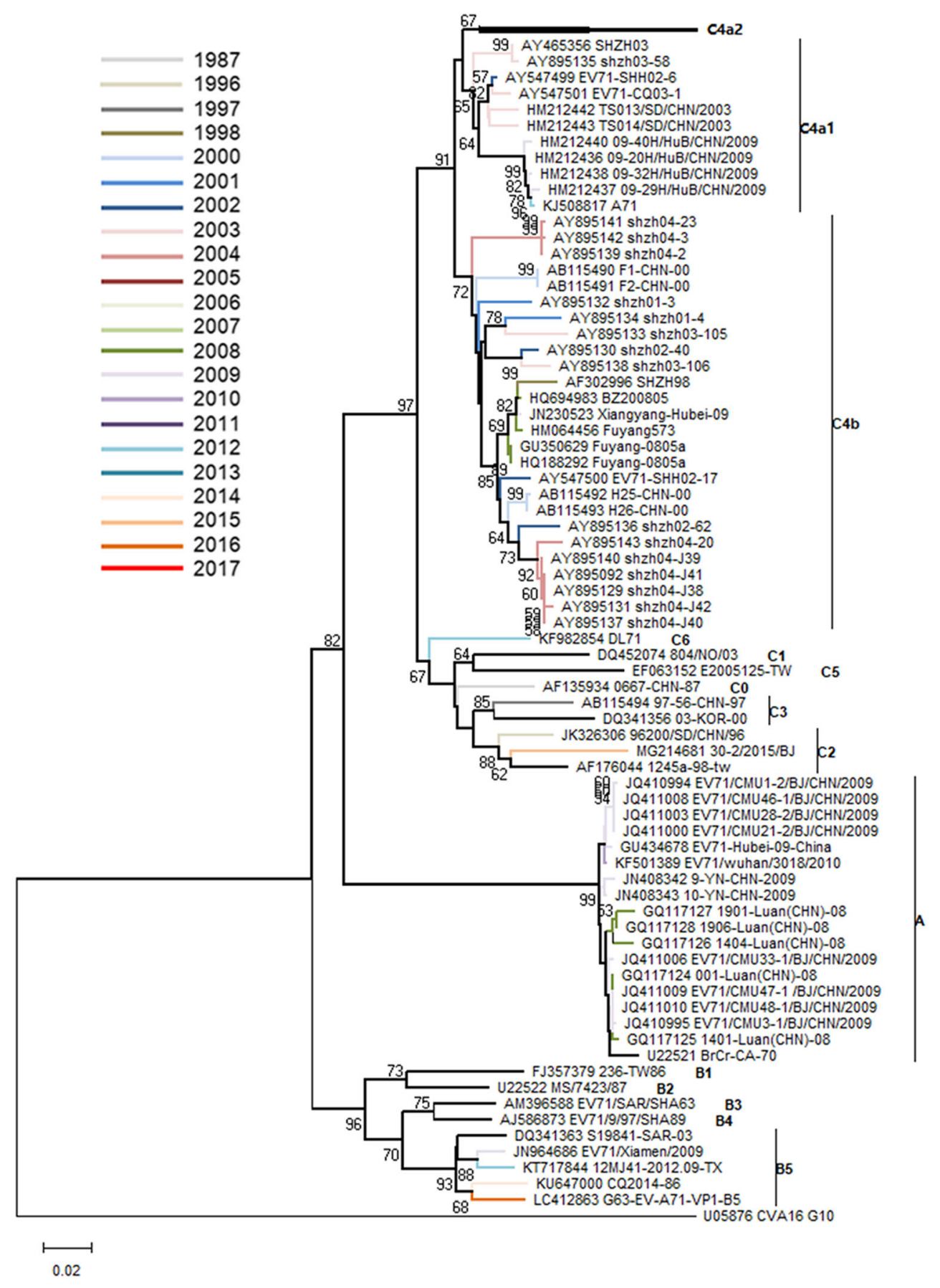

\section{Time and space distribution of subgenotypes of EV-} A71 in mainland China

To describe the prevalence of EV-A71 subgenotypes in mainland China, the information including place and time of isolation and subgenotypes of 3037 EV-A71 strains was recorded (Fig. 2). In 1987-1997, EV-A71 exhibited an extremely low prevalence in mainland China. Since 1998, C4 EV-A71 strains emerged, which became the predominant subgenotype. Three shifts in the predominant subgenotype between clades $\mathrm{C} 4 \mathrm{~b}$ and $\mathrm{C} 4 \mathrm{a} 2$ were observed. $\mathrm{C} 4 \mathrm{~b}$ was the predominant subgenotype in 1998-2002 and 2004. The first shift from $\mathrm{C} 4 \mathrm{~b}$ to $\mathrm{C} 4 \mathrm{a} 2$ was observed in 2003. The second shift from $\mathrm{C} 4 \mathrm{a} 2$ to $\mathrm{C} 4 \mathrm{~b}$ was observed in 2004, followed by the third shift from $\mathrm{C} 4 \mathrm{~b}$ to $\mathrm{C} 4 \mathrm{a} 2$ in 2005 . Since then, $\mathrm{C} 4 \mathrm{a} 2$ has been the predominant subgenotype. Moreover, different from the sporadic emerging subgenotypes $\mathrm{C} 0, \mathrm{C} 2, \mathrm{C} 3$, and $\mathrm{B} 5$, the genotype A EV-A71 strains co-circulated with both $\mathrm{C} 4 \mathrm{~b}$ and $\mathrm{C} 4 \mathrm{a} 1$ in Central China in 2009, and the subgenotype C6 EV-A71 strain co-circulated with the re-emerged C4a1 in Northeast China in 2012. 

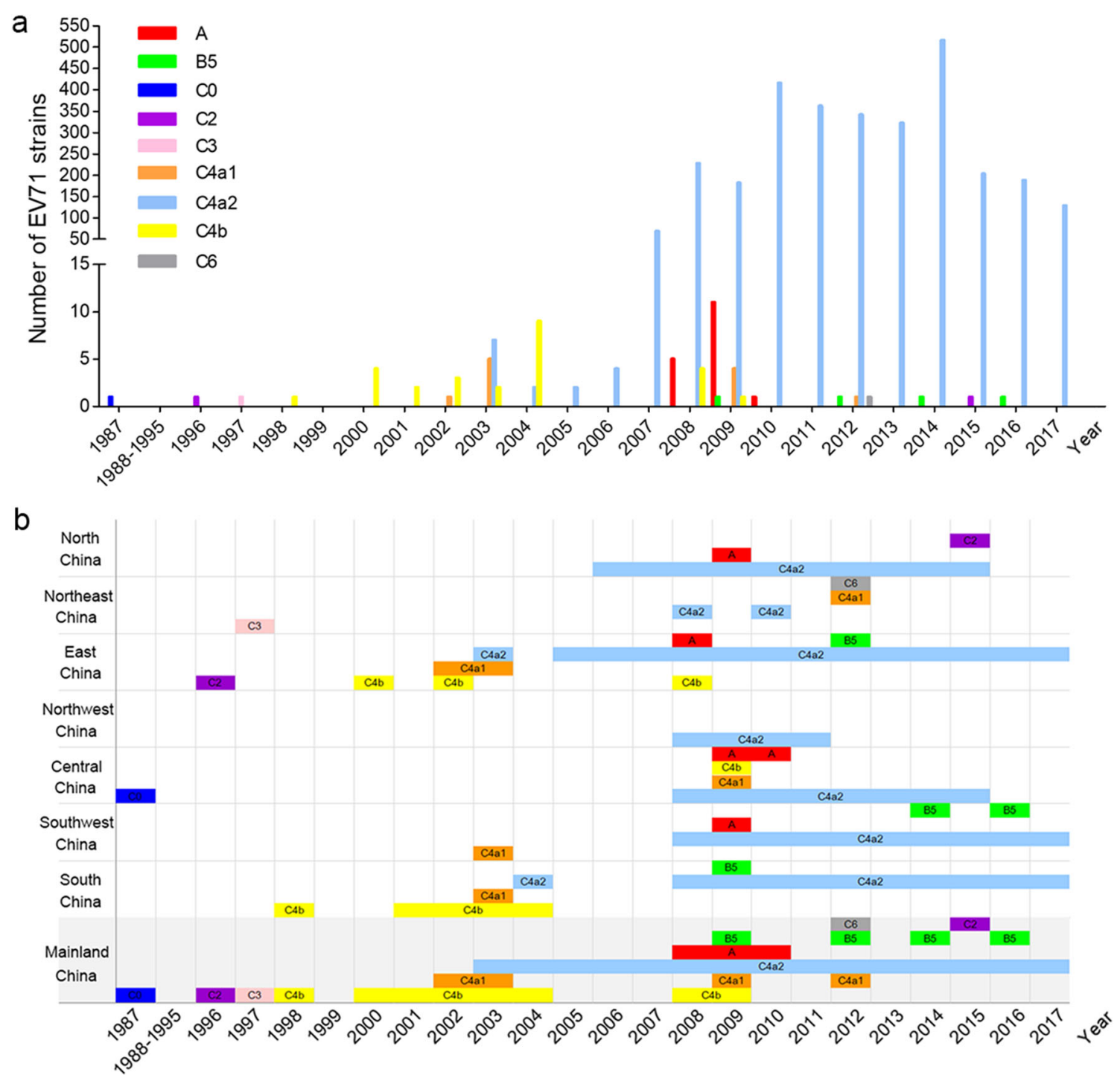

Fig. 2 Temporal and geographical distribution of EV-A71 subgenotypes in mainland China during 1987-2017. a Temporal distribution of EVA71 subgenotypes was analyzed by isolated time of EV-A71 strains. b

\section{Recombination analysis of Chinese C4 EV-A71 strains and other EV-A strains}

To characterize the genomic features of the predominant subgenotype C4 EV-A71 strains, the complete genome sequences of 3 EV-A71 strains, BZ200805 (HQ694983, C4b, 2008), SHZH03 (AY465356, C4a1, 2003), and 160-50 (MG875331, C4a2, 2016), were selected as the query sequences for similarity and bootscan analysis (Fig. 3). Similarity plot analyses indicated that C4 EV-A71 strains (including $\mathrm{C} 4 \mathrm{~b}, \mathrm{C} 4 \mathrm{a} 1$, and $\mathrm{C} 4 \mathrm{a} 2$ ) exhibited the highest degree of similarity to all C EV-A71 strains in the P1 region; however, in the $\mathrm{P} 2$ and $\mathrm{P} 3$ regions, they contained an unidentified sequence that was apparently not related to C EV-A71 strains (Fig. 3a, c, and e). Two different similarity patterns for C4 EVA71 strains were observed in the $\mathrm{P} 2$ and $\mathrm{P} 3$ regions. One
Geographical distribution of EV-A71 subgenotypes was analyzed by the isolated places of EV-A71 strains

pattern showed that C4 EV-A71 strains resembled CVA4, CVA5, CVA14, and CVA16 in the region from 3700 to $6000 \mathrm{bp}$, and in the region after $6000 \mathrm{bp}$, they still resembled CVA4, CVA14, and CVA16 but away from CVA5. The other pattern showed that C4 EV-A71 strains shared high similarity to all B EV-A71 strains in the region from 3700 to $5650 \mathrm{bp}$, while in the region after $5650 \mathrm{bp}$, they still shared high similarity to B3 EV-A71 strains but away from B1, B2, B4, and B5 EV-A71 strains.

Moreover, the bootscan analyses suggested possible recombination events with at least two recombinant breakpoints on $\mathrm{C} 4 \mathrm{EV}-\mathrm{A} 71$ corresponding to the $2 \mathrm{~A} / 2 \mathrm{~B}$ junction and $3 \mathrm{C} /$ 3D junction (Fig. 3b, d, and f). C4 EV-A71 strains contained alternative regions that were closely related to B EV-A71 strains, CVA5, CVA4, CVA14, and CVA16 strains in the region between the $2 \mathrm{~A} / 2 \mathrm{~B}$ and $3 \mathrm{C} / 3 \mathrm{D}$ junctions. And in the 

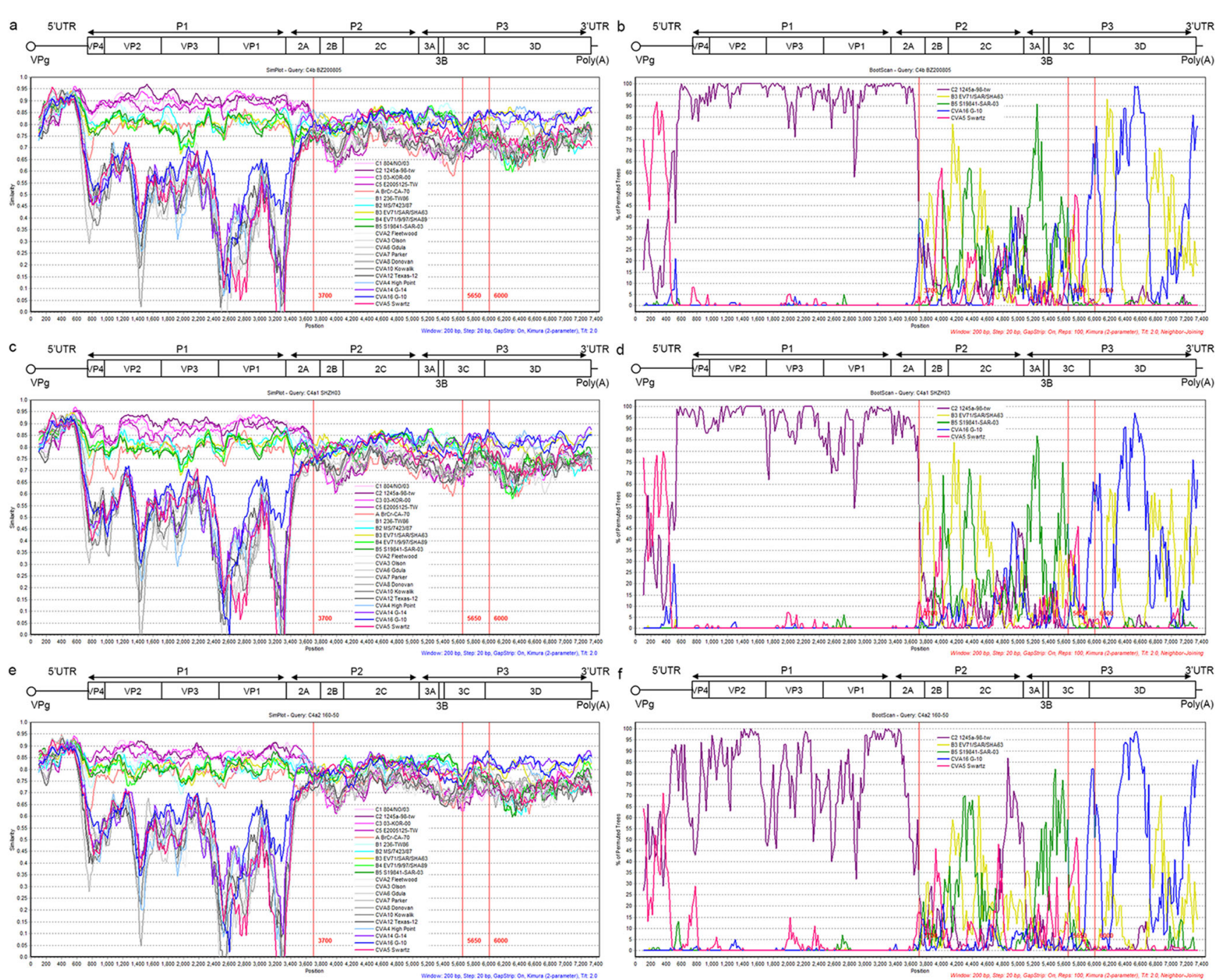

Fig. 3 Recombination analysis of the complete genome of C4 EV-A71 strains in mainland China. The genome sequences of BZ200805, subgenotype C4b (a, b), SHZH03, subgenotype C4a1 (c, d), and 16050 , subgenotype $\mathrm{C} 4 \mathrm{a} 2(\mathbf{e}, \mathbf{f})$ were used as query sequences in the

region after the 3C/3D junction, C4 EV-A71 strains were more closely related to B3 EV-A71, CVA4, CVA14, and CVA16 strains.

\section{Recombination analysis of Chinese B5, C2, C6 EV-A71 strains and other EV-A strains}

The genomic characteristics of EV-A71 strains belonging to $\mathrm{B} 5, \mathrm{C} 2$, and $\mathrm{C} 6$ were also analyzed. The complete genome sequences of EV-A71 strains CQ2014-86 (KU647000, B5, 2014), 30-2/2015/BJ (MG214681, C2, 2015), and DL71 (KF982854, C6, 2012) were selected as the query sequences for the similarity and bootscan analysis (Fig. 4).

For B5 strain CQ2014-86 (Fig. 4a and b), the sequence before the $2 \mathrm{~A} / 2 \mathrm{~B}$ junction (nearby $3750 \mathrm{bp}$ ) resembled $\mathrm{C}$ EV-A71 strains, while the fragment (3750-5662 bp) was more similar to C4 EV-A71, CVA4, CVA5, CVA14, and

similarity plot (left panels) and bootscan analysis (right panels). The structural map of EV-A71 genome was displayed upon each panel. The possible crossover breakpoints marked with red vertical line was showed in the analysis

CVA16 strains than C1-C3 and C5 EV-A71 strains. The fragment (5662-6500 bp) of CQ2014-86 shared higher similarity to CVA3, CVA5, CVA6, CVA10, and CVA12 strains than C EV-A71 strains. The sequence after 6500 bp of CQ2014-86 was more similar to $\mathrm{C} 1-\mathrm{C} 3$ and $\mathrm{C} 5 \mathrm{EV}-\mathrm{A} 71$ strains than $\mathrm{C} 4$ EV-A71 strains. Two recombination events possibly occurred in B5 EV-A71 strain: one occurred between C4 EV-A71 and CVA3, CVA5, CVA6, CVA10, and CVA12 strains with breakpoint located in the $3 \mathrm{C}$ gene, and the other occurred between C1-C3 and C5 EV-A71 and CVA4, CVA5, CVA14, and CVA16 strains with two breakpoints located in the $2 \mathrm{~A} / 2 \mathrm{~B}$ junction and $3 \mathrm{C}$ gene.

For C2 strain 30-2/2015/BJ (Fig. 4c and d), the results provided strong evidence that it might be a recombinant virus between C4 EV-A71 and CVA8 strains with two breakpoints located in the $5^{\prime} \mathrm{UTR}(550 \mathrm{bp}$ ) and $2 \mathrm{~A} / 2 \mathrm{~B}$ junction ( $3750 \mathrm{bp}$ ). The sequence $(550-3750 \mathrm{bp})$ of $30-2 / 2015 / \mathrm{BJ}$ shared the 


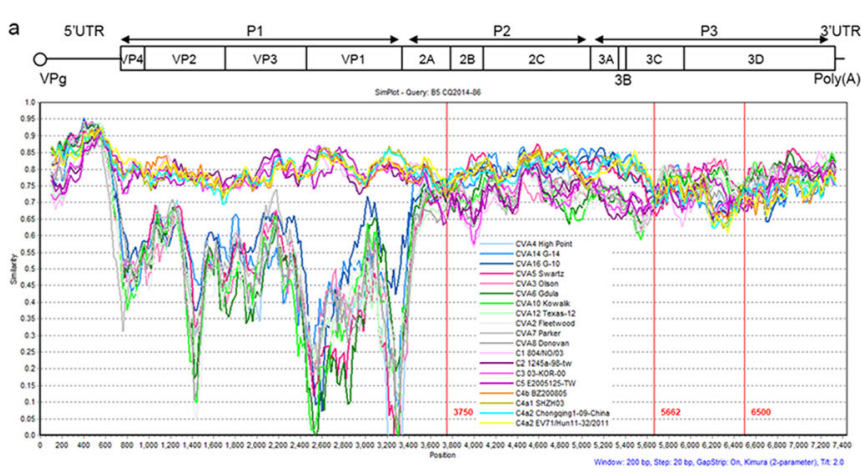

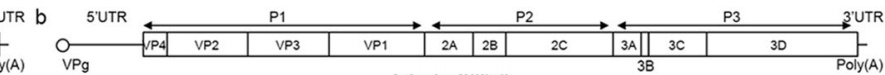
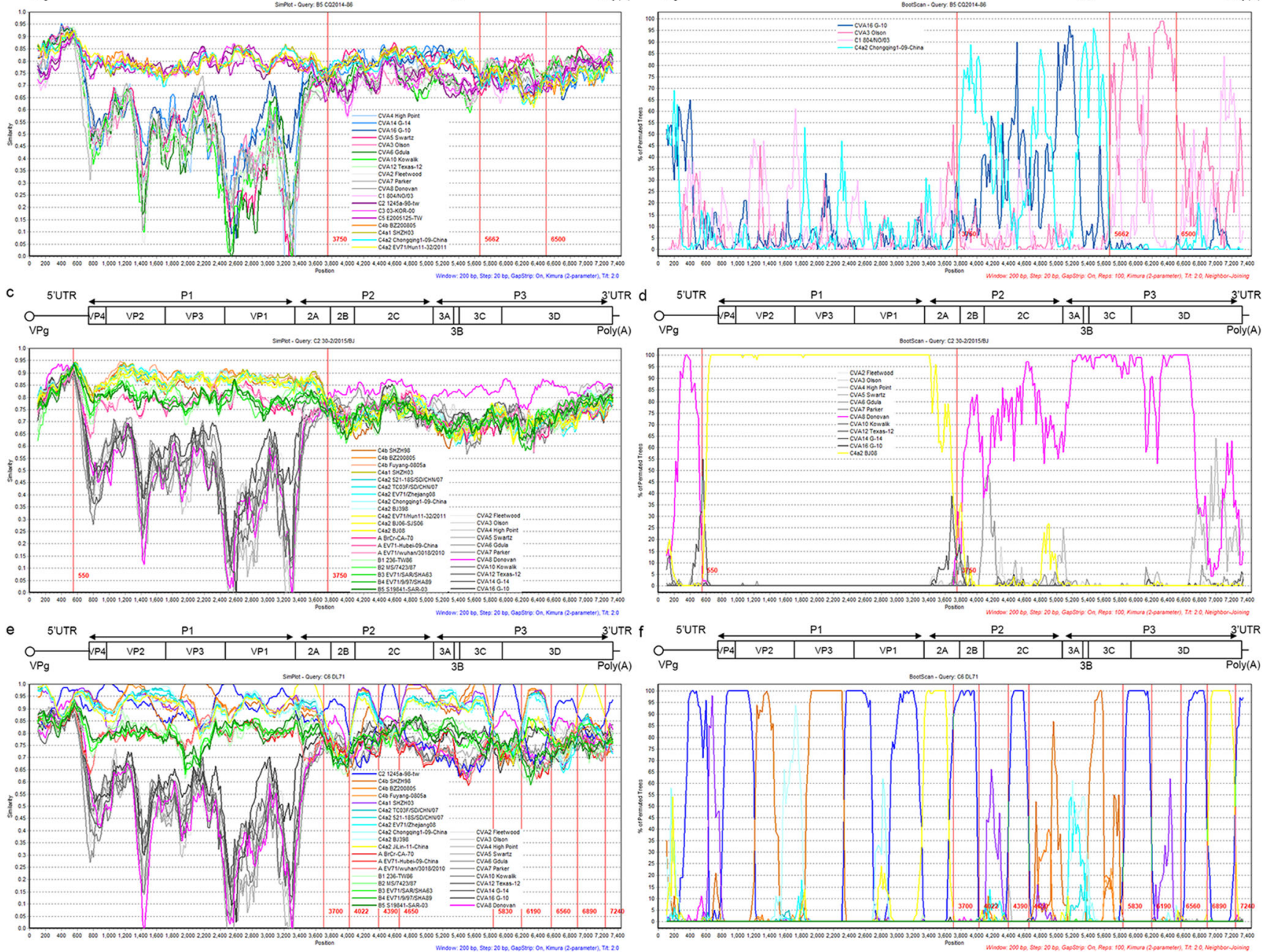

Fig. 4 Emergence of EV-A71 subgenotypes B5, C2, and C6 by recombination with other EV-A strains. The whole genome sequences of CQ2014-86, subgenotype B5 (a, b), 30-2/2015/BJ, subgenotype C2 (c, d), and DL71, subgenotype C6 (e, f) were used in the similarity plot (left

highest similarity to C4 EV-A71 strains, while the 5'UTR (1$550 \mathrm{bp}$ ) and the sequence after $3750 \mathrm{bp}$ of $30-2 / 2015 / \mathrm{BJ}$ shared the highest similarity to CVA8 strain.

For C6 strain DL71 (Fig. 4e and f), the similarity plot analysis exhibited an irregular helix-like recombination on DL71 formed by the winding of the genome sequences of C4 and C2 EV-A71 strains with multiple breakpoints. In the $\mathrm{P} 1$ region, DL71 shared higher similarity to $\mathrm{C} 4$ and $\mathrm{C} 2 \mathrm{EV}-$ A71 strains $(>80 \%)$ than B EV-A71 strains $(65-85 \%)$ and other EV-A strains $(<75 \%)$. In the $\mathrm{P} 2$ and $\mathrm{P} 3$ regions, 4 fragments (4022-4390 bp, 4650-5830 bp, 6190-6560 bp, and 6890-7240 bp) of DL71 shared higher similarity to C4 EVA71 strains $(>80 \%)$ than C2 EV-A71 strain $(<80 \%)$, while other 5 fragments (3700-4022-bp, 4390-4650-bp, 58306190-bp, 6560-6890-bp, and 7240-bp 3'UTR) of DL71 shared higher similarity to C2 EV-A71 strain (>80\%) than C4 EV-A71 strains $(<80 \%)$.

panels) and bootscan analysis (right panels). The structural map of EVA71 genome was displayed upon each panel. The possible crossover breakpoints marked with red vertical line was showed in the analysis

\section{Discussion}

In this study, the genetic diversity of EV-A71 strains in mainland China was well demonstrated by the molecular epidemiology and recombination analyses. Besides the predominant subgenotypes $\mathrm{C} 4$, some other sporadic subgenotypes A, B5, $\mathrm{C} 0, \mathrm{C} 2$, and $\mathrm{C} 3$ and the new subgenotype $\mathrm{C} 6$ emerged in mainland China. The first C2 EV-A71 strain 96200/SD/ $\mathrm{CHN} / 96$ in mainland China isolated from an acute flaccid paralysis case shared high identity in VP1 with the second C2 EV-A71 strain 30-2/2015/BJ (Tao et al., 2012; Li et al., 2018). The later $C 2$ strain was isolated from a child who had traveled back from Thailand, where $\mathrm{C} 2$ was commonly detected ( $\mathrm{Li}$ et al., 2018). The $\mathrm{C} 2$ strains in mainland China might be imported from other countries or areas. In mainland China, the first B5 strain was detected in 2009, and then 3 strains were isolated in 2012, 2014, and 2016. Subgenotype 
B5 strains were first detected in Taiwan in 1998 (Wu et al., 2013) and then circulated widely in the Asia-Pacific region. In 2009, subgenotype B5 strains simultaneously circulated in Japan, Malaysia, and Taiwan. Frequent international travel might promote the import of sporadic viruses into mainland China. Moreover, a new finding in this study was the emergence of C6 EV-A71. One orphan strain DL71 was clustered into the new subgenotype C6 different from the previously known subgenotypes $\mathrm{C} 0-\mathrm{C} 5$ in genotype $\mathrm{C}$. The results suggested that several subgenotypes of EV-A71 had existed in China. Thus, the emergence and importation of new genotypes or subgenotypes of EV-A71 should be continuously monitored (Li et al., 2018; Noisumdaeng et al., 2018).

Genetic recombination plays important roles in the genetic variation and evolution of viruses. Several studies have provided evidence of recombinant events in EV-A71, including intratypic recombination between different subgenotypes of EV-A71 and intertypic recombination between EV-A71 and other EV-A strains (Zhang et al., 2011; Zhang et al., 2013; Yoke-Fun and AbuBakar, 2006; Wang et al., 2012). However, the query sequences of EV-A71 selected in recombination analysis in previous studies were mostly the consensus sequences or the oldest strains within different subgenotypes (Zhang et al., 2013), and few sequences of newly isolated EV-A71 strains were used as the query sequences. In the present study, the complete genome sequences of the latest EV-A71 strains within each subgenotype were used as the query sequences. Our comprehensive recombination analysis exhibited two patterns of recombination in C4 EV-A71 strains (Fig. 3). We found that intertypic recombination of $\mathrm{C} 4$ sequences occurred between the structural genes of C EV-A71 and the nonstructural genes of CVA4, CVA5, CVA14, and CVA16, which was consistent with the findings in previous reports (Zhang et al., 2011; Zhang et al., 2013). However, the evidence of intratypic recombination between EV-A71 genotypes C and B was not sufficient (Zhang et al., 2013). In addition to intertypic recombination, our study also showed evidence of intratypic recombination between EV-A71 genotypes $\mathrm{C}$ and B. The fragment (3700-5650 bp) of C4 EV-A71 resembled B EV-A71, and this recombination pattern was also found in other C4 EV-A71 strains isolated in Thailand (Noisumdaeng et al., 2018; Mauleekoonphairoj et al., 2015), Taiwan (Huang et al., 2008), and Hong Kong (Yip et al., 2013). Moreover, we found interestingly that except for the fragment (3700-5650 bp) of C4 being closer to B3, the sequence after 5650 bp of C4 EV-A71 still shared high similarity to B3 but away from B1, B2, B4, and $\mathrm{B} 5$. A phenomenon in a previous study that the $\mathrm{P} 2$ and $\mathrm{P} 3$ regions of B3 EV-A71 had high similarity to CVA4, CVA14, and CVA16 (Yoke-Fun and AbuBakar, 2006) also supported our results. Thus, there was strong evidence to support the conclusion of the intertypic recombination between genotype C EV-A71 strains and other EV-A strains and the intratypic recombination between EV-A71 genotypes C and B. Several studies renamed this "double-recombinant" C4 EV-A71 strain genotype D (Yip et al., 2010; Yip et al., 2013).

The genomic characteristics of EV-A71 strains belonging to $\mathrm{B} 5, \mathrm{C} 2$, and $\mathrm{C} 6$ in mainland China were also analyzed (Fig. 4). The recombination manner of $\mathrm{C} 2 \mathrm{EV}-\mathrm{A} 71$ strain $30-2 /$ 2015/BJ in mainland China was similar to other C2 EV-A71 strains isolated in Malaysia and Taiwan (Yoke-Fun and AbuBakar, 2006), which resembled C4 EV-A71 strains in the $\mathrm{P} 1$ region and resembled the prototype strain of CVA8 in the 5'UTR, P2, and P3 regions. The B5 EV-A71 strain CQ2014-86 was closer to C EV-A71 strains in the P1 region but contained an unidentified sequence in the $\mathrm{P} 2$ and $\mathrm{P} 3$ regions. Given the global distribution of EV-A71 subgenotypes C2 and B5 (Mizuta et al., 2014; Wu et al., 2013), the sporadic $\mathrm{C} 2$ and B5 strains that emerged in mainland China were most likely to have been imported from other countries and areas. An interesting finding was that DL71 (Dalian City, 2012) was a recombinant between the dominant subgenotype $\mathrm{C} 4$ and the sporadic subgenotype C2 EV-A71 strains with a helix-like recombination manner. The details of recombination events in DL71 needed further studies.

The HFMD outbreaks and EV-A71 circulation in mainland China provided conditions for C6 EV-A71 emergence. Firstly, frequent outbreaks of HFMD occurred in Dalian City with the highest number of cases in 2012 during the period of 2010-2013 (Gao et al., 2016; Wang et al., 2019). Secondly, as one parent strain of C6, C4 was the single predominant subgenotype in mainland China with much genetic diversity (including $\mathrm{C} 4 \mathrm{a} 1, \mathrm{C} 4 \mathrm{a} 2$, and $\mathrm{C} 4 \mathrm{~b}$ ). Thirdly, as another parent strain of $\mathrm{C} 6, \mathrm{C} 2$ has been widely detected in many countries for several years (World Health Organization [WHO], 2011). The importation of $\mathrm{C} 2$ from neighboring countries led to its sporadic circulation in mainland China. Thus, the prevalence of HFMD, the genetic diversity of the predominant subgenotype $\mathrm{C} 4$, and the sporadic circulation of $\mathrm{C} 2$ might together promote the recombination and emergence of C6 EV-A71. Moreover, no evidence was reported about the clinical symptoms and disease severity of DL71. Based on reviewing the potential virulence determinants of EV-A71 in previous reports (Huang et al., 2015; Kung et al., 2010; Li et al., 2016; Li et al., 2011; Liu et al., 2014b; Ma et al., 2018; Meng and Kwang, 2014; Wen et al., 2013; Yuan et al., 2015), the nucleotides and amino acids located at these virulence sites in DL71 were analyzed (Table S3). In DL71 genome, two nucleotides and four amino acids might be with high virulence at six sites, while six nucleotides and twelve amino acids might be with low virulence at eighteen sites. In fact, the pathogenicity of EV-A71 was very complex and influenced by many factors including virus virulence and host immunity. It was difficult to judge the pathogenicity of C6 EV-A71. This was one limitation in the study.

Our present findings that recombination breakpoints in the EV-A71 genome were frequently located in the non-structural 
regions 5'UTR, P2, and P3 but rarely in the structural region P1 were consistent with previous reports (Zhang et al., 2011; Yip et al., 2010; Noisumdaeng et al., 2018; Zhang et al., 2013; Yoke-Fun and AbuBakar, 2006; Huang et al., 2008; Zhang et al., 2010; Wang et al., 2012; Li et al., 2012; Yip et al., 2013; Liu et al., 2014a; Mauleekoonphairoj et al., 2015). On one hand, the P1 region of EV-A71 maintained a high degree of similarity within EV-A71 strains, but away from other EV-A strains, possibly because of the need for structural constraint to ensure the proper assembly of viral capsid (Wang et al., 2012). While recombination occurred in the structural genes, it might produce nonviable or unstable progeny viruses that could not survive (Stanway et al., 1986). On the other hand, the reason for the non-structural regions $\mathrm{P} 2$ and $\mathrm{P} 3$ as the hot spots for recombination in EV-A71 was that the P2 and P3 regions of EV-A shared more homology than the structural region (Wang et al., 2012). The acquisition of non-structural genes encoding replicative components such as protease, helicase, and polymerase from B EV-A71 strains and other EV-A strains (CVA4, CVA5, CVA14, and CVA16) in C4 EV-A71 strains might provide evolutionary advantages for the "double-recombinant" strains (Yip et al., 2013). In addition, the inconsistent topology of phylogenetic trees suggested that it was not sufficient to genotype the recombinant viruses by VP1 alone. Given that these recombinant viruses might be a threat to public health in mainland China, it was still worthwhile to closely monitor the prevalence of EV-A71 and the emergence of new subgenotypes by performing extensive studies on the viral complete genome.

Supplementary Information The online version contains supplementary material available at https://doi.org/10.1007/s10123-021-00164-2.

Acknowledgments We thank all the staffs engaged in the study of hand, foot, and mouth disease throughout China for their enormous contributions.

Data and materials availability The datasets generated during and/or analyzed during the current study are available from the corresponding author on reasonable request.

Code availability MEGA-X software RRID: SCR_000667.

Author's contribution Yongjuan Liu contributed to the study conception and design. Material preparation and data collection were performed by Jingyi Zhou, Yingying Shi, Li Miao, and Chunyan Zhang. Data analysis was performed by Jingyi Zhou and Yongjuan Liu. The first draft of the manuscript was written by Jingyi Zhou. The manuscript review and editing were performed by Yongjuan Liu. All authors read and approved the final manuscript.

Funding This work was funded by the Natural Science Foundation of Jiangsu Province (BK20180269), the Natural Science Foundation of Hubei Province (2018CFB254), Wuhan COVID-19 Emergency Research Project (EX20D04), the Technology Bureau and Social Development Project of Lianyungang City (SH1525), the Youth
Talents Hansoh Foundation of Lianyungang No. 1 People's Hospital (QN160204, QN160103), and Dr. Scientific Research Start-up foundation of Lianyungang No. 1 People's Hospital (BS1507).

\section{Declarations}

Ethics approval This article does not contain any studies with human or animal subjects performed by any of the authors.

Consent to participate Not applicable.

Consent for publication Not applicable.

Conflict of interest The authors declare no competing interests.

\section{References}

Bessaud M, Razafindratsimandresy R, Nougairede A, Joffret M, Deshpande J, Dubot-Peres A, Heraud J, de Lamballerie X, Delpeyroux F, Bailly J (2014) Molecular comparison and evolutionary analyses of VP1 nucleotide sequences of new African human enterovirus 71 isolates reveal a wide genetic diversity. PLoS One 9: e90624

Chen G, Sun L, Fang Q, Song L, Shi Y, Zhang J, Ma W, Su B (2019) Advances in research on risk factors and prevention and control measures of severe hand, foot and mouth disease. J Prev Med Public Health 30:94-98

Deshpande J, Nadkarni S, Francis P (2003) Enterovirus 71 isolated from a case of acute flaccid paralysis in India represents a new genotype. Curr Sci 84:1350-1353

Gao Y, Liu Y, Sun D (2016) Analysis of 28479 cases of hand-foot-andmouth disease in Dalian from 2010 to 2013. Chinese Pediatrics of Integrated Traditional And Western Medicine 8:217-219

Huang SC, Hsu YW, Wang HC, Huang SW, Kiang D, Tsai HP, Wang SM, Liu CC, Lin KH, Su IJ, Wang JR (2008) Appearance of intratypic recombination of enterovirus 71 in Taiwan from 2002 to 2005. Virus Res 131:250-259

Huang SW, Tai CH, Fonville JM, Lin CH, Wang SM, Liu CC, Su IJ, Smith DJ, Wang JR (2015) Mapping Enterovirus A71 antigenic determinants from viral evolution. J Virol 89:11500-11506

Kung YH, Huang SW, Kuo PH, Kiang D, Ho MS, Liu CC, Yu CK, Su IJ, Ren WJ (2010) Introduction of a strong temperature-sensitive phenotype into enterovirus 71 by altering an amino acid of virus 3D polymerase. Virology 396:1-9

Li R, Zou Q, Chen L, Zhang H, Wang Y (2011) Molecular analysis of virulent determinants of enterovirus 71. PLoS One 6:e26237

Li J, Huo X, Dai Y, Yang Z, Lei Y, Jiang Y, Li G, Zhan J, Zhan F (2012) Evidences for intertypic and intratypic recombinant events in EV71 of hand, foot and mouth disease during an epidemic in Hubei Province, China, 2011. Virus Res 169:195-202

Li P, Yue Y, Song N, Li B, Meng H, Yang G, Li Z, An L, Qin L (2016) Genome analysis of enterovirus 71 strains differing in mouse pathogenicity. Virus Genes 52:161-171

Li J, Li Y, Zhang S, Ma H, Liu X, Liang Z, Zhang W, Jing H, Du Y, Yang Y, Huo D, Chen L, Wang Q (2018) Analysis of an imported subgenotype C2 strain of human Enterovirus 71 in Beijing, China, 2015. Front Microbiol 9:2337

Lin K, Zhang H, Bai W, Zhao Y, Liu H, Huang X, Yang Z, Ma S (2017) Complete genome sequence of an Enterovirus 71 strain isolated from the cerebrospinal fluid of a child with severe hand-foot-andmouth disease in Yunnan, China, 2013. Genome Announc 5: e00274-e00217 
Liu W, Wu S, Xiong Y, Li T, Wen Z, Yan M, Qin K, Liu Y, Wu J (2014a) Co-circulation and genomic recombination of coxsackievirus A16 and enterovirus 71 during a large outbreak of hand, foot, and mouth disease in Central China. PLoS One 9:e96051

Liu Y, Fu C, Wu S, Chen X, Shi Y, Zhou B, Zhang L, Zhang F, Wang Z, Zhang Y, Fan C, Han S, Yin J, Peng B, Liu W, He X (2014b) A novel finding for enterovirus virulence from the capsid protein VP1 of EV71 circulating in mainland China. Virus Genes 48:260-272

Ma H, Lu C, Tsao K, Shih H, Cheng A, Huang L, Chang L (2018) Association of EV71 3C polymorphisms with clinical severity. J Microbiol Immunol Infect 51:608-613

Majumdar M, Sharif S, Klapsa D, Wilton T, Alam M, Fernandez-Garcia M, Rehman L, Mujtaba G, McAllister G, Harvala H, Templeton K, Mee E, Asghar H, Ndiaye K, Minor P, Martin J (2018) Environmental surveillance reveals complex Enterovirus circulation patterns in human populations. Open Forum Infect Dis 5:ofy250

Mauleekoonphairoj J, Vongpunsawad S, Puenpa J, Korkong S, Poovorawan Y (2015) Complete genome sequence analysis of enterovirus 71 isolated from children with hand, foot, and mouth disease in Thailand, 2012-2014. Virus Genes 51:290-293

Meng T, Kwang J (2014) Attenuation of human enterovirus 71 highreplication-fidelity variants in AG129 mice. J Virol 88:5803-5815

Mizuta K, Aoki Y, Matoba Y, Yahagi K, Itagaki T, Katsushima F, Katsushima Y, Ito S, Hongo S, Matsuzaki Y (2014) Molecular epidemiology of enterovirus 71 strains isolated from children in Yamagata, Japan, between 1990 and 2013. J Med Microbiol 63: 1356-1362

Noisumdaeng P, Sangsiriwut K, Prasertsopon J, Klinmalai C, Payungporn S, Mungaomklang A, Chokephaibulkit K, Buathong R, Thitithanyanont A, Puthavathana P (2018) Complete genome analysis demonstrates multiple introductions of enterovirus 71 and coxsackievirus A16 recombinant strains into Thailand during the past decade. Emerg Microbes Infect 7:214

Saxena V, Sane S, Nadkarni S, Sharma D, Deshpande J (2015) Genetic diversity of enterovirus A71, India. Emerg Infect Dis 21:123-126

Schmidt NJ, Lennette EH, Ho HH (1974) An apparently new enterovirus isolated from patients with disease of the central nervous system. J Infect Dis 129:304-309

Simmonds P, Gorbalenya A, Harvala H, Knowles N, Lindberg A, Oberste M, Palmenberg A, Reuter G, Skern T, Tapparel C, Wolthers K, Woo P, Zell R (2020) Recommendations for the nomenclature of enteroviruses and rhinoviruses. Arch Virol 165:793797

Stanway G, Hughes PJ, Westrop GD, Evans DM, Dunn G, Minor PD, Schild GC, Almond JW (1986) Construction of poliovirus intertypic recombinants by use of cDNA. J Virol 57:1187-1190

Tao Z, Wang H, Xu A (2012) Identification of a C2 subgenogroup strain of enterovirus 71 in a retrospective study in Shandong Province, China, from 1990 to 2010. J Clin Microbiol 50:1823-1824

Vakulenko Y, Deviatkin A, Lukashev A (2019) Using statistical phylogenetics for investigation of Enterovirus 71 genotype A reintroduction into circulation. Viruses 11:895

Wang H, Li Y (2019) Recent progress on functional genomics research of Enterovirus 71. Virol Sin 34:9-21

Wang X, Zhu C, Bao W, Zhao K, Niu J, Yu XF, Zhang W (2012) Characterization of full-length enterovirus 71 strains from severe and mild disease patients in northeastern China. PLoS One 7:e32405

Wang Z, Wang L, Yu W, Sun Y, Mao L, Yao W (2019) Epidemiologic characteristics and pathogen surveillance of hand, foot and mouth disease in Liaoning Province, 2008-2017. Chin J Exp Clin Virol 33: 6-9

Wen H, Si L, Yuan X, Hao S, Gao F, Chu F, Sun C, Wang Z (2013) Complete genome sequencing and analysis of six enterovirus 71 strains with different clinical phenotypes. Virol J 10:115

World Health Organization [WHO] (2011) A guide to clinical management and public health response for hand, foot and mouth disease (HFMD). Reginal emerging disease intervention, Geneva:WHO

Wu JS, Zhao N, Pan H, Wang CM, Wu B, Zhang HM, He HX, Liu D, Amer S, Liu SL (2013) Patterns of polymorphism and divergence in the VP1 gene of enterovirus 71 circulating in the Asia-Pacific region between 1994 and 2013. J Virol Methods 193:713-728

Yip CC, Lau SK, Zhou B, Zhang MX, Tsoi HW, Chan KH, Chen XC, Woo PC, Yuen KY (2010) Emergence of enterovirus 71 "doublerecombinant" strains belonging to a novel genotype D originating from southern China: first evidence for combination of intratypic and intertypic recombination events in EV71. Arch Virol 155: $1413-1424$

Yip CC, Lau SK, Lo JY, Chan KH, Woo PC, Yuen KY (2013) Genetic characterization of EV71 isolates from 2004 to 2010 reveals predominance and persistent circulation of the newly proposed genotype $\mathrm{D}$ and recent emergence of a distinct lineage of subgenotype $\mathrm{C} 2$ in Hong Kong. Virol J 10:222

Yoke-Fun C, AbuBakar S (2006) Phylogenetic evidence for inter-typic recombination in the emergence of human enterovirus 71 subgenotypes. BMC Microbiol 6:74

Yuan S, Li G, Wang Y, Gao Q, Wang Y, Cui R, Altmeyer R, Zou G (2015) Identification of positively charged residues in Enterovirus 71 capsid protein VP1 essential for production of infectious particles. J Virol 90:741-752

Zhang Y, Tan XJ, Wang HY, Yan DM, Zhu SL, Wang DY, Ji F, Wang XJ, Gao YJ, Chen L, An HQ, Li DX, Wang SW, Xu AQ, Wang ZJ, Xu WB (2009) An outbreak of hand, foot, and mouth disease associated with subgenotype C4 of human enterovirus 71 in Shandong, China. J Clin Virol 44:262-267

Zhang Y, Zhu Z, Yang W, Ren J, Tan X, Wang Y, Mao N, Xu S, Zhu S, Cui A, Zhang Y, Yan D, Li Q, Dong X, Zhang J, Zhao Y, Wan J, Feng Z, Sun J, Wang S, Li D, Xu W (2010) An emerging recombinant human enterovirus 71 responsible for the 2008 outbreak of hand foot and mouth disease in Fuyang City of China. Virol J 7:94

Zhang Y, Wang J, Guo W, Wang H, Zhu S, Wang D, Bai R, Li X, Yan D, Wang H, Zhang Y, Zhu Z, Tan X, An H, Xu A, Xu W (2011) Emergence and transmission pathways of rapidly evolving evolutionary branch $\mathrm{C} 4 \mathrm{a}$ strains of human enterovirus 71 in the Central Plain of China. PLoS One 6:e27895

Zhang Y, Tan X, Cui A, Mao N, Xu S, Zhu Z, Zhou J, Shi J, Zhao Y, Wang X, Huang X, Zhu S, Zhang Y, Tang W, Ling H, Xu W (2013) Complete genome analysis of the $\mathrm{C} 4$ subgenotype strains of enterovirus 71: predominant recombination $\mathrm{C} 4$ viruses persistently circulating in China for 14 years. PLoS One 8:e56341

Zhao S (2019) Progress in diagnosis and treatment of severe hand, foot and mouth disease. Chinese Journal of Applied Clinical Pediatrics 34:1689-1692

Publisher's note Springer Nature remains neutral with regard to jurisdictional claims in published maps and institutional affiliations. 